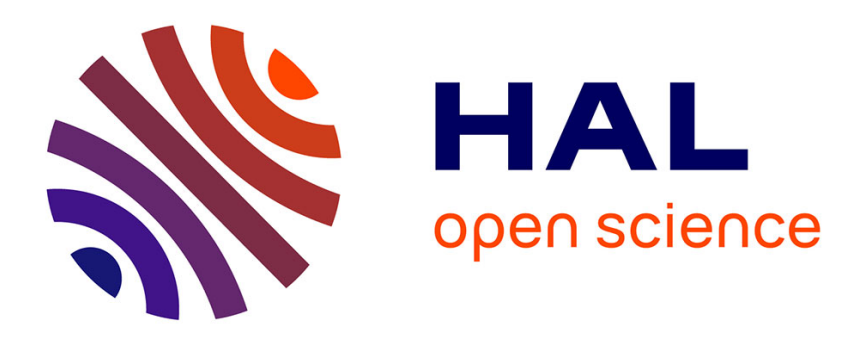

\title{
Recovery of steady periodic wave profiles from pressure measurements at the bed
}

Didier Clamond, Adrian Constantin

\section{To cite this version:}

Didier Clamond, Adrian Constantin. Recovery of steady periodic wave profiles from pressure measurements at the bed. Journal of Fluid Mechanics, 2013, 10.1017/jfm.2012.490 . hal-01302715

\section{HAL Id: hal-01302715 \\ https://hal.science/hal-01302715}

Submitted on 20 Dec 2018

HAL is a multi-disciplinary open access archive for the deposit and dissemination of scientific research documents, whether they are published or not. The documents may come from teaching and research institutions in France or abroad, or from public or private research centers.
L'archive ouverte pluridisciplinaire HAL, est destinée au dépôt et à la diffusion de documents scientifiques de niveau recherche, publiés ou non, émanant des établissements d'enseignement et de recherche français ou étrangers, des laboratoires publics ou privés. 


\title{
Recovery of steady periodic wave profiles from pressure measurements at the bed
}

\author{
D. Clamond ${ }^{1}$ and A. Constantin ${ }^{2,3,} \dagger$ \\ ${ }^{1}$ Laboratoire J.-A. Dieudonné, Université de Nice - Sophia Antipolis, Parc Valrose, \\ 06108 Nice CEDEX 2, France \\ ${ }^{2}$ Department of Mathematics, King's College London, Strand, London WC2R 2LS, UK \\ ${ }^{3}$ Faculty of Mathematics, University of Vienna, Nordbergstrasse 15, 1090 Vienna, Austria
}

We derive an equation relating the pressure at the flat bed and the profile of an irrotational steady water wave, valid for all classical solutions of the governing equations for water waves. This permits the recovery of the surface wave from pressure measurements at the bed. Although we focus on periodic waves, the extension to solitary waves is straightforward. We illustrate the usefulness of the equation beyond the realm of linear theory by investigating the regime of shallow-water waves of small amplitude and by presenting a numerical example.

Key words: surface gravity waves, waves/free-surface flows

\section{Introduction}

Within the framework of incompressible, homogeneous and inviscid flow modelling of water waves, the pressure acts as a Lagrange multiplier to enforce the divergencefree constraint on the velocity field. The pressure plays an important role in establishing various qualitative properties of travelling water waves in irrotational flow, e.g. in the description of the particle trajectories beneath the waves (Constantin 2006; Constantin \& Strauss 2010), and in showing that an overhanging wave profile is not possible (Spielvogel 1970; Varvaruca 2006). The pressure is also essential in quantitative studies because often in practice the elevation of a surface water wave is determined from pressure data obtained at the sea bed (Kuo \& Chiu 1994; Baquerizo \& Losada 1995; Tsai et al. 2005).

The standard approach - used, for instance, in open-ocean buoys (see the discussion in Oliveras et al. 2012) - consists of assuming that the hydrostatic approximation

$$
\eta(x)=\mathfrak{p}(x) / g-d, \quad x \in \mathbb{R},
$$

is sufficiently accurate. Here the water density $\rho$ is constant, $g$ is the (constant) acceleration due to gravity (directed downwards), and

$$
\mathfrak{p}(x)=\left[P(x,-d)-P_{\text {atm }}\right] / \rho
$$

is the normalized relative pressure evaluated at the flat bed $y=-d$, with $d$ representing the average depth of the fluid, $P(x,-d)$ being the pressure as a function

$\dagger$ Email address for correspondence: adrian.constantin@kcl.ac.uk 
of the spatial variable $x$, evaluated at the flat bed, $P_{a t m}$ the (constant) atmospheric pressure at the water surface, and $x \mapsto \eta(x)$ the profile of the steady surface water wave travelling at constant speed $c>0$. However, the discussion by Bishop \& Donelan (1987) shows that, even for waves of moderate amplitude, prediction errors exceeding $15 \%$ frequently occur if one relies on (1.1). These inaccuracies are due to the fact that (1.1) ignores even linear wave effects.

Within the linear regime of water waves of small amplitude in finite depth, one can derive (see e.g. Escher \& Schlurmann 2008) the so-called transfer-function formula

$$
\eta(x)=\cosh (k d)[\mathfrak{p}(x)-g d] / g, \quad x \in \mathbb{R},
$$

where $k=2 \pi / L$ is the wavenumber corresponding to the wavelength $L>0$. Notice that the hydrostatic formula (1.1) is recovered in the limit $k d \rightarrow 0$ of (1.3). The benefit of the simplicity of the linear formula (1.3) is offset by the fact that for waves of moderate amplitude it often overestimates the wave height by more than $10 \%$, cf. the experimental data provided by Tsai et al. (2005).

These considerations motivate the quest for a reconstruction formula that accounts for nonlinear effects and that is thus applicable to waves of moderate and large amplitude. Recently, a nonlinear non-local equation relating the dynamic pressure on the bed and the wave profile was obtained without approximation from the governing equations (Deconinck et al. 2011; Oliveras et al. 2012). The equation reads

$$
\sqrt{\frac{c^{2}-2 g \eta(x)}{1+\left[\eta_{x}(x)\right]^{2}}}=\sum_{k=-\infty}^{\infty} \mathrm{e}^{\mathrm{i} k x} \hat{\theta}_{k} \cosh (k[\eta(x)+d]), \quad x \in \mathbb{R},
$$

where $\eta_{x}=\mathrm{d} \eta / \mathrm{d} x$ and

$$
\hat{\theta}_{k}=\frac{1}{2 \pi} \int_{0}^{2 \pi} \mathrm{e}^{-\mathrm{i} k x} \sqrt{c^{2}-2 \mathfrak{p}(x)+2 g d} \mathrm{~d} x, \quad k \in \mathbb{Z} .
$$

The entangled character of (1.4) is a considerable impediment in the pursuit of an in-depth analysis. Our aim is to present a more expedient approach that applies to all smooth steady waves.

We first establish the existence of a holomorphic function $\mathfrak{P}$ on the fluid domain, whose restriction to the flat bed has zero imaginary part and real part $\mathfrak{p}$. The wave amplitude $\eta_{0}>0$ is determined as the unique fixed point of the contraction

$$
s \mapsto(\operatorname{Re} \mathfrak{P})(0, s) / g-d,
$$

and the surface wave profile $x \mapsto \eta(x)$ is given by the unique solution of the ordinary differential equation

$$
\eta_{x}=\frac{(\operatorname{Im} \mathfrak{P})(x, \eta)}{B-g \eta-(\operatorname{Re} \mathfrak{P})(x, \eta)+g d},
$$

with initial data $\eta(0)=\eta_{0}$. Here $B>0$ is the Bernoulli constant, determined by requiring that the solution to (1.7) has zero mean. The fact that the right-hand side of (1.7) is a smooth and bounded function of $x$ and $\eta$ is a byproduct of our approach. In contrast to the implicit nonlinear equation (1.4), a standard iterative procedure solves (1.6)-(1.7).

Throughout this paper, we are concerned with periodic waves but the same approach works for solitary waves. In this context, let us point out that recently 
an explicit formula providing a parametric representation of irrotational solitary wave profiles in terms of the pressure on the flat bed was obtained by Constantin (2012). We do not pursue the periodic analogue of that procedure here. Instead, our alternative approach is applicable in the physical variables. This presents a definite advantage from the practical point of view since a periodic analogue of the approach pursued in Constantin (2012) would involve a conformal hodograph transform that is computationally and experimentally less acquiescent.

\section{Preliminaries}

In a frame of reference moving at the constant wave speed $c$, the flow beneath a travelling wave reduces to a steady motion with respect to the moving coordinate system. Let $(x, y)$ be a Cartesian coordinate system moving with the wave, $x$ being the horizontal coordinate and $y$ the upward vertical coordinate. Let $(u(x, y), v(x, y))$ be the velocity field in the moving frame. The wave is $L=(2 \pi / k)$-periodic in the $x$-direction, and we denote by $y=-d, y=\eta(x)$ and $y=0$ the equations of the bottom, of the free surface and of the mean water level, respectively. The last equation expresses the fact that $\langle\eta\rangle=0$ for the smooth $L$-periodic wave profile $\eta$, where $\langle\cdot\rangle$ is the Eulerian average operator over one wavelength,

$$
\langle\eta\rangle \equiv \frac{k}{2 \pi} \int_{-\pi / k}^{\pi / k} \eta(x) \mathrm{d} x=0 .
$$

The flow is governed by the balance between the restoring gravity force and the inertia of the system. With constant density $\rho>0$, the equation of mass conservation and Euler's equations are

$$
u_{x}+v_{y}=0
$$

and

$$
u u_{x}+v u_{y}=-P_{x} / \rho, \quad u v_{x}+v v_{y}=-P_{y} / \rho-g,
$$

respectively, where $P(x, y)$ denotes the pressure. We are investigating gravity water waves, so that the assumption of a homogeneous inviscid flow is appropriate. The effect of surface tension being negligible, on the free surface we must have

$$
P=P_{\text {atm }} \quad \text { on } y=\eta(x),
$$

where $P_{a t m}$ is the (constant) atmospheric pressure. The dynamic boundary condition decouples the motion of the water from that of the air above it. The fact that the free surface and the rigid bed are interfaces is captured by the kinematic boundary conditions

$$
v=u \eta_{x} \quad \text { on } y=\eta(x)
$$

and

$$
v=0 \quad \text { on } y=-d,
$$

respectively, while the irrotational character of the flow is ensured by requiring

$$
u_{y}=v_{x} .
$$

Equations (2.2)-(2.7) are the governing equations for irrotational travelling water waves in a moving frame. We refer to Constantin (2011) for an in-depth discussion of the rationale for the underlying physical assumptions. 
Throughout this paper, we call a Stokes wave a smooth solution to the governing equations for which $\eta, u, v, P$ are all periodic in the $x$-variable, with the functions $\eta, u, P$ even and $v$ odd in the $x$-variable. Moreover, the wave profile $\eta$ should be strictly monotonic between consecutive crests and troughs, and symmetric. (Our definition of a Stokes wave includes all wavelength over depth ratios.) The existence theory for Stokes waves is based on bifurcation theory, see the discussions by Toland (1996) and Constantin (2011). Some of the above assumptions are not restrictive. For example, a continuously differentiable wave profile must actually be real-analytic and the velocity components have harmonic extensions across the free surface (see Toland 1996; Constantin \& Escher 2011). Moreover, the free surface has to be the graph of a function (Toland 1996) and the symmetry of the free surface is guaranteed if the wave profile is monotonic between crests and troughs (Okamoto \& Shōji 2001), in which case $\eta_{x}<0$ for $x \in(0, L / 2)$, if $x=0$ is the location of the wave crest. The only Stokes waves for which the free surface is not a continuously differentiable curve are the waves of greatest height, for which the curve is real-analytic except at the crest where it is just continuous with a corner containing an inner angle of $2 \pi / 3$ (cf. the discussion in Toland 1996). Throughout this paper, we are only concerned with smooth Stokes waves. In this case the flow presents no stagnation points, that is,

$$
u(x, y)<0 \text { for } x \in \mathbb{R},-d \leqslant y \leqslant \eta(x) .
$$

For the Stokes wave of greatest height the inequality (2.8) breaks down at the wave crest, where $u=0$, and this fact is intimately connected with the loss of regularity (Toland 1996). We refrain from discussing the Stokes wave of greatest height.

Let $\phi$ and $\psi$ be the velocity potential and the stream function, respectively, such that

$$
u=\phi_{x}=\psi_{y}, \quad v=\phi_{y}=-\psi_{x} .
$$

It is convenient to introduce the complex potential and the complex velocity

$$
f \equiv \phi+\mathrm{i} \psi, \quad w \equiv u-\mathrm{i} v
$$

respectively, that are holomorphic functions of the complex variable $z=x+\mathrm{i} y$, with $f=f(z)$ and $w=\mathrm{d} f / \mathrm{d} z$. The equation of mass conservation for a homogeneous fluid and the irrotational character of the flow are encompassed in the relations (2.9) together with the requirement that $\phi$ and $\psi$ are harmonic functions throughout the fluid domain. The Euler equation can be expressed by means of the Bernoulli condition:

$$
2 p+2 g y+u^{2}+v^{2}=B, \quad x \in \mathbb{R},-d \leqslant y \leqslant \eta(x),
$$

for some constant $B$, where

$$
p(x, y)=\left[P(x, y)-P_{a t m}\right] / \rho, \quad x \in \mathbb{R},-d \leqslant y \leqslant \eta(x),
$$

is the normalized relative pressure. From (2.1), (2.4) and (2.11), we get

$$
B=\left\langle u_{s}^{2}+v_{s}^{2}\right\rangle
$$

where $u_{s}$ and $v_{s}$ denote the restrictions of $u$ and $v$ to the free surface, respectively. On the other hand, applying the divergence theorem to the vector field $\left(-2 u v, u^{2}-v^{2}\right)$ in the domain $\{(x, y):-\pi / k \leqslant x \leqslant \pi / k,-d \leqslant y \leqslant \eta(x)\}$ and taking into account (2.5), (2.6) and the periodicity condition in the $x$-variable, we obtain

$$
\left\langle u_{s}^{2}+v_{s}^{2}\right\rangle=\left\langle u_{b}^{2}\right\rangle
$$


where $u_{b}$ denotes the restriction of $u$ to the flat bed $y=-d$. The relations (2.11), (2.13) and (2.14) yield

$$
\langle\mathfrak{p}\rangle=g d,
$$

where $\mathfrak{p}(x)=p(x,-d)$ is the normalized relative pressure at the bed. The relation (2.15) is very important for practical applications because it can be used to recover the average water depth from the measured pressure at the bed.

\section{Recovery of the wave profile}

Instead of dealing with the complex potential $f$ or with the complex velocity $w$, it is advantageous to use the holomorphic function $w^{2}$. The function

$$
\mathfrak{P}(z) \equiv \frac{1}{2} B+g d-\frac{1}{2} w^{2}(z)=\frac{1}{2} B+g d-\frac{1}{2}\left(u^{2}-v^{2}\right)+\mathrm{i} u v
$$

is holomorphic in the fluid domain $\{(x, y): x \in \mathbb{R},-d \leqslant y \leqslant \eta(x)\}$, and its restriction to the flat bed $y=-d$ has zero imaginary part and real part $\mathfrak{p}$, i.e.

$$
\mathfrak{p}(x)=\mathfrak{P}(x-\mathrm{i} d)=g d+\frac{1}{2}\left(B-u_{b}^{2}\right) .
$$

Thus $\mathfrak{p}$ determines $\mathfrak{P}$ uniquely throughout the fluid domain. Note that $p$ defined in (2.12) coincides with the real part $(\operatorname{Re} \mathfrak{P})$ only on $y=-d$ because the former is not a harmonic function in the fluid domain (Constantin 2006; Constantin \& Strauss 2010). Using (2.5) and (2.11) on the free surface $y=\eta(x)$, where $p=0$ owing to (2.4) and (2.12), we have

$$
\begin{aligned}
\left(u_{s}-\mathrm{i} v_{s}\right)^{2} & =\left(1-\mathrm{i} \eta_{x}\right)^{2} u_{s}^{2}=\left(1+\eta_{x}^{2}\right) u_{s}^{2}\left(1-\mathrm{i} \eta_{x}\right) /\left(1+\mathrm{i} \eta_{x}\right) \\
& =\left(u_{s}^{2}+v_{s}^{2}\right)\left(1-\mathrm{i} \eta_{x}\right) /\left(1+\mathrm{i} \eta_{x}\right)=(B-2 g \eta)\left(1-\mathrm{i} \eta_{x}\right) /\left(1+\mathrm{i} \eta_{x}\right) .
\end{aligned}
$$

Multiplying this relation by $\left(1+\mathrm{i} \eta_{x}\right)$ and using (3.1), we obtain at once

$$
g \eta\left(1-\mathrm{i} \eta_{x}\right)+\mathrm{i} B \eta_{x}=[\mathfrak{P}(x+\mathrm{i} \eta)-g d]\left(1+\mathrm{i} \eta_{x}\right) .
$$

The real and imaginary parts of (3.4) give two equations for $\eta$ :

$$
\begin{aligned}
g \eta & =(\operatorname{Re} \mathfrak{P})_{s}-g d-\eta_{x}(\operatorname{Im} \mathfrak{P})_{s}, \\
B \eta_{x}-g \eta \eta_{x} & =\left[(\operatorname{Re} \mathfrak{P})_{s}-g d\right] \eta_{x}+(\operatorname{ImP})_{s},
\end{aligned}
$$

where, as above, the subscript ' $s$ ' denotes the evaluation at the free surface $y=\eta(x)$. Using (3.1), we can see that (3.5)-(3.6) are precisely

$$
\begin{aligned}
g \eta & =\frac{1}{2}\left[B-u_{s}^{2}-v_{s}^{2}\right], \\
(B-g \eta) \eta_{x} & =\frac{1}{2}\left[B+u_{s}^{2}+v_{s}^{2}\right] \eta_{x} .
\end{aligned}
$$

Thus, both are ensured by the validity of the Bernoulli condition (2.11) on the free surface. For a Stokes wave of non-zero amplitude, it is known that $\eta_{x} \neq 0$ between consecutive crests and troughs (Constantin 2006; Constantin \& Strauss 2010). Therefore, not only does (3.7) imply (3.8), but also (3.8) ensures the validity of (3.7) between consecutive crests and troughs, and by continuous extension everywhere.

For the recovery of the surface wave profile $\eta$, given the function $\mathfrak{p}$, one can proceed as follows. Owing to (2.15), the pressure $p$ at the bed $y=-d$ has the Fourier series expansion

$$
\mathfrak{p}(x)=g d+\sum_{\substack{n=-\infty \\ n \neq 0}}^{\infty} \mathfrak{p}_{n} \mathrm{e}^{\mathrm{i} n k x}
$$


with $\mathfrak{p}_{-n}=\overline{\mathfrak{p}}_{n}$ for $n \neq 0$ since $\mathfrak{p}$ is real. From (3.9) we get

$$
\mathfrak{P}(z)=\mathfrak{P}(x, y)=g d+\sum_{\substack{n=-\infty \\ n \neq 0}}^{\infty} \mathfrak{p}_{n} \mathrm{e}^{\mathrm{i} n k[x+\mathrm{i}(y+d)]},
$$

showing that $\mathfrak{P}$ can be easily obtained from $\mathfrak{p}$. The validity of the above series expansion throughout a horizontal strip that contains the fluid domain in its interior can be proved by adapting to the flat-bed context the approach pursued in Plotnikov \& Toland (2002) for a fluid of infinite depth. This ensures that even though the complex velocity field has no holomorphic extension to the entire complex plane, it extends analytically to a strip of the form $\{(x, y) \in \mathbb{R}:-\eta(0)-2 d-\varepsilon<y<\eta(0)+\varepsilon\}$ for some $\varepsilon>0$.

We now evaluate (3.5) at the wave crest located at $x=0$, where $\eta_{x}=0$, obtaining the implicit equation

$$
\eta_{0}=(\operatorname{Re} \mathfrak{P})\left(0, \eta_{0}\right) / g-d .
$$

(A similar relation holds at the trough, of course.) The crest height $\eta_{0}$ is obtained as the unique solution to (3.11). The function on the right-hand side of (3.11) is a contraction, so that its unique fixed point can be obtained by a standard iterative procedure; see the discussion in $\S 5$ and the numerical example in $\S 6$. With $\eta_{0}$ determined by solving (3.11) iteratively, we now solve the ordinary differential equation

$$
\eta_{x}=\frac{(\operatorname{Im} \mathfrak{P})(x, \eta)}{B-g \eta-(\operatorname{Re} \mathfrak{P})(x, \eta)+g d},
$$

with initial data $\eta(0)=\eta_{0}$. Equation (3.12) is merely a re-expression of (3.6). We claim that the right-hand side of (3.12) is smooth, so that the solution can be obtained by the standard iterative procedure implemented on the formulation of (3.12) as a nonlinear integral equation; see the discussion in $\S 5$. To prove our claim, note that by (3.1) we have

$$
B-g \eta-(\operatorname{Re} \mathfrak{P})_{s}+g d=\frac{1}{2} B-g \eta+\frac{1}{2} u_{s}^{2}-\frac{1}{2} v_{s}^{2}=u_{s}^{2}>0,
$$

owing to (2.8) and (2.11) and the fact that $p=0$ along $y=\eta(x)$. The above procedure is contingent upon the determination of the Bernoulli constant $B$, obtained by imposing the constraint of zero mean to the solution $\eta$ to (3.12) with initial data $\eta(0)=\eta_{0}$; see the discussion in $\$ 5$.

For the practical identification of the location of the wave crest from recorded values of $\mathfrak{p}$, the results by Constantin \& Strauss (2010) ensure that the maximum of $p$ in the fluid domain is attained on the bed beneath the wave crest, the minimum of $p$ is attained all along the free surface, and $\mathfrak{p}$ is strictly monotonic between consecutive crests and troughs, its minimum being attained beneath the wave trough. These facts ensure that recorded data on $\mathfrak{p}$ determine the location of the wave crest and of the wave trough.

\section{Perturbation expansion examples}

In this section we illustrate the applicability of our approach in various regimes.

\subsection{Hydrostatic approximation}

With $P(x, y)=P_{a t m}-g y$ throughout the fluid we have $\mathfrak{p}(x)=g d$. This yields $\mathfrak{P}(z)=g d$, so that (3.11) becomes $\eta_{0}=0$ and (3.12) becomes $\eta_{x}=0$. We conclude that $\eta \equiv 0$, a confirmation that we have a flat horizontal free surface. 


\subsection{Linear theory of waves of small amplitude}

Within this framework, in terms of the small amplitude parameter $\varepsilon>0$, we have

$$
\begin{aligned}
\mathfrak{p}(x) & =g d+\varepsilon g d \operatorname{sech}(k d) \cos (k x)+O\left(\varepsilon^{2}\right), \\
\eta(x) & =\varepsilon d \cos (k x)+O\left(\varepsilon^{2}\right), \\
B & =(g / k) \tanh (k d)+O\left(\varepsilon^{2}\right),
\end{aligned}
$$

cf. the discussion by Constantin (2011). Substituting $x$ by $x+\mathrm{i}(y+d)$ in (4.1) and splitting the real and imaginary parts, we get after some trivial algebra

$$
\frac{\mathfrak{P}(x, y)}{g d}=1+\varepsilon \frac{\cosh (k(y+d))}{\cosh (k d)} \cos (k x)-\mathrm{i} \varepsilon \frac{\sinh (k(y+d))}{\cosh (k d)} \sin (k x)+O\left(\varepsilon^{2}\right) .
$$

Therefore (3.11) and (3.12) take the form

$$
\left.\begin{array}{l}
\eta_{0}=\frac{\varepsilon d \cosh \left(k\left(\eta_{0}+d\right)\right)}{\cosh (k d)}+O\left(\varepsilon^{2}\right), \\
\eta_{x}=-\frac{\varepsilon k d \sinh (k(\eta+d)) \sin (k x)}{\sinh (k d)}+O\left(\varepsilon^{2}\right),
\end{array}\right\}
$$

respectively. The solution of the first equation is of the form

$$
\eta_{0}=\varepsilon d+O\left(\varepsilon^{2}\right)
$$

so that $\eta=O(\varepsilon)$. In view of (4.3), this allows us to write the above differential equation as

$$
\eta_{x}=-\varepsilon k d \sin (k x)+O\left(\varepsilon^{2}\right) .
$$

This yields (4.2). Notice the validity of (1.3) if we ignore terms of $O\left(\varepsilon^{2}\right)$.

\subsection{Cnoidal shallow-water theory}

The Korteweg-de Vries (KdV) equation arises as a weakly nonlinear model for the propagation of shallow-water waves of small amplitude. Its periodic travelling waves can be expressed using the elliptic functions sn, $\mathrm{cn}$ and $\mathrm{dn}$, and the complete elliptic integrals of the first and second kind $K$ and $E$, depending on the parameter $m \in[0,1]$. For example, we have the expressions (Clamond 1998, 1999, 2003)

$$
\begin{aligned}
\eta(x) & =\eta_{0}\left\{\operatorname{dn}^{2}(\kappa x \mid m)-E(m) / K(m)\right\} /\{1-E(m) / K(m)\}, \\
u(x,-d) & =u_{0}\left\{\mathrm{dn}^{2}(\kappa x \mid m)-E(m) / K(m)\right\}-c,
\end{aligned}
$$

for the free surface and for the horizontal fluid velocity on the flat bed, respectively. Here, $\kappa=2 K(m) / L$ is related to the wavenumber $k=2 \pi / L$, while $\eta_{0}>0, u_{0}>0$ and $c>0$ are parameters, $c$ being the wave phase velocity observed in the reference frame in which the horizontal velocity has average zero at the bed. Note that the expression of $u(x,-d)$ used by Clamond $(1999,2003)$ and Henry (2009) are in that frame of reference, while (4.9) is expressed in the frame following the wave. Note also that for $m \ll 1$

$$
u(x,-d)=\frac{1}{2} m u_{0} \cos (2 \kappa x)-c+O\left(m^{2}\right),
$$

thus recovering the approximation of the previous subsection.

The KdV-regime is characterized by

$$
\eta_{0} / d=O\left(\delta^{2}\right), \quad u_{0} / c=O\left(\delta^{2}\right) \quad \text { and } \quad \kappa d=O(\delta),
$$


where $\delta \ll 1$ is a dimensionless parameter characterizing the ratio of depth over typical wavelength. The approximations (4.8) and (4.9) neglect terms of $O\left(\delta^{4}\right)$. An approximation of the same order for the square of the horizontal velocity at the bed is

$$
u^{2}(x,-d)=c^{2}-2 c u_{0}\left\{\operatorname{dn}^{2}(\kappa x \mid m)-E(m) / K(m)\right\}+O\left(\delta^{4}\right) .
$$

We obtain the approximation

$$
\mathfrak{p}(x)=\frac{1}{2}\left(B-c^{2}\right)+g d+c u_{0}\left\{\operatorname{dn}^{2}(\kappa x \mid m)-E(m) / K(m)\right\}+O\left(\delta^{4}\right),
$$

for the pressure at the bed. The conditions (2.13) and (2.14) now yield

$$
B=c^{2}+O\left(\delta^{4}\right),
$$

so that

$$
\mathfrak{p}(x) \approx g d+A\left\{\mathrm{dn}^{2}(\kappa x \mid m)-E(m) / K(m)\right\},
$$

with $A=c u_{0}$. Once the parameters $m, \kappa$ and $A$ have been obtained (e.g. by fitting experimental data), the function $\mathfrak{P}$ is easily obtained by replacing $x$ by $x+\mathrm{i}(y+d)$ in the right-hand side of (4.15). Splitting the expression into real and imaginary parts, one obtains

$$
\begin{aligned}
& \operatorname{Re} \mathfrak{P}=g d+A\left\{\frac{d_{1}^{2}(x) c_{2}^{2}(y) d_{2}^{2}(y)-m^{2} s_{1}^{2}(x) c_{1}^{2}(x) s_{2}^{2}(y)}{\left[c_{2}^{2}(y)+m s_{1}^{2}(x) s_{2}^{2}(y)\right]^{2}}-\frac{E(m)}{K(m)}\right\}, \\
& \operatorname{Im} \mathfrak{P}=-2 m A \frac{s_{1}(x) c_{1}(x) d_{1}(x) s_{2}(y) c_{2}(y) d_{2}(y)}{\left[c_{2}^{2}(y)+m s_{1}^{2}(x) s_{2}^{2}(y)\right]^{2}},
\end{aligned}
$$

with $\left\{s_{1} ; c_{1} ; d_{1}\right\}(x)=\{\mathrm{sn} ; \mathrm{cn} ; \operatorname{dn}\}(\kappa x \mid m)$ and $\left\{s_{2} ; c_{2} ; d_{2}\right\}(y)=\{\mathrm{sn} ; \mathrm{cn} ; \operatorname{dn}\}[\kappa(y+d) \mid$ $1-m]$.

The approximations (4.16) and (4.17) can be used to compute the free surface from the relations (3.11) and (3.12). While it was demonstrated by Clamond (1999, 2003) that renormalized cnoidal waves are uniformly valid for all depths, and that they are also quite accurate for large waves, we focus on the KdV-regime. The Taylor expansion

$$
\mathfrak{P}(x+\mathrm{i} y)=\exp \left[\mathrm{i}(y+d) \partial_{x}\right] \mathfrak{p}(x)
$$

around $y=-d$ (Clamond 1999) allows us, in the KdV-regime, where $\partial_{x}=O(\delta)$ and $\eta=O\left(\delta^{2}\right)$, to use the approximation

$$
\mathfrak{P}(x+\mathrm{i} \eta)=\mathfrak{p}(x)+\mathrm{i} d \mathfrak{p}_{x}(x)+O\left(\delta^{4}\right) .
$$

Thence

$$
\begin{aligned}
& (\operatorname{Re} \mathfrak{P})(x, \eta(x))=g d+A\left\{\operatorname{dn}^{2}(\kappa x \mid m)-E(m) / K(m)\right\}+O\left(\delta^{4}\right), \\
& (\operatorname{Im} \mathfrak{P})(x, \eta(x))=-2 m \kappa d A \operatorname{sn}(\kappa x \mid m) \operatorname{cn}(\kappa x \mid m) \operatorname{dn}(\kappa x \mid m)+O\left(\delta^{5}\right) .
\end{aligned}
$$

These approximations enable us to write (3.12) as

$$
\eta_{x}=-2 m \kappa d(A / B) \operatorname{sn}(\kappa x \mid m) \operatorname{cn}(\kappa x \mid m) \operatorname{dn}(\kappa x \mid m)+O\left(\delta^{5}\right)
$$

and integration on $[0 ; x]$ yields

$$
\eta(x)-\eta_{0}=d(A / B)\left[\operatorname{dn}^{2}(\kappa x \mid m)-1\right]+O\left(\delta^{4}\right) .
$$


Using (3.11), we determine the wave amplitude

$$
\eta_{0}=A[1-E(m) / K(m)] / g .
$$

Finally, the last relation needed to completely recover the solution (4.8) is obtained by enforcing (2.1). Indeed, (2.1) and (4.22) lead to

$$
\eta_{0}=d(A / B)[1-E(m) / K(m)] .
$$

Eliminating $A$ and $B$ between (4.22), (4.23) and (4.24) yields (4.8), up to $O\left(\delta^{4}\right)$.

\section{The general reconstruction procedure}

Knowing $\mathfrak{p}$, we determine $\mathfrak{P}$ using (3.10). Denoting

$$
F(y)=(\operatorname{Re} \mathfrak{P})(0, y) / g-d, \quad y \in\left[-d, \eta_{0}\right],
$$

we can recast (3.11) as $\eta_{0}$ being a fixed point of $F$. In view of (3.1) and (2.11), we have that $\mathfrak{P}=p+g(y+d)$ all along the crest line $x=0$, where $v=0$. Thus

$$
F(y)=y+p(0, y) / g=\left[B-u^{2}(0, y)\right] /(2 g), \quad y \in\left[-d, \eta_{0}\right] .
$$

The results by Constantin \& Strauss (2010) ensure that

$$
u_{y}(0, y)>0>p_{y}(0, y), \quad y \in\left[-d, \eta_{0}\right]
$$

for every smooth Stokes wave. The above inequality combined with (2.8) and (5.2) shows that $F$ is a contraction. Consequently, starting at any $y_{0} \in[-d ; 0]$, the sequence of iterates $\left\{y_{n}\right\}_{n \geqslant 1}$, given by $y_{n}=F\left(y_{n-1}\right)$ for $n \geqslant 1$, converges to the unique fixed point $\eta_{0}$ of $F$ (Coppel 1965). It is of interest to point out that since the function $p$ attains its minimum throughout the fluid domain only at the free surface (Constantin \& Strauss 2010), where $p=0$ in view of (2.4) and (2.12), the first equality in (5.2) implies that the sequence $\left\{y_{n}\right\}_{n \geqslant 0}$ is strictly increasing. This iterative procedure determines the wave amplitude $\eta_{0}$, while $B$ and $\eta(x)$ remain to be found.

Let us now observe that by (2.11) and (3.1), we have for all $-d \leqslant y \leqslant \eta(x)$ that

$$
\begin{gathered}
B-g y-(\operatorname{Re} \mathfrak{P})(x, y)+g d=p(x, y)+u^{2}(x, y) \geqslant u^{2}(x, y) \\
\geqslant u^{2}\left(0, \eta_{0}\right)=B-g \eta_{0}-(\operatorname{Re} \mathfrak{P})\left(0, \eta_{0}\right)+g d>0,
\end{gathered}
$$

in view of the results by Constantin \& Strauss (2010). Therefore, for $b$ such that

$$
b>g\left(\eta_{0}-d\right)+(\operatorname{Re} \mathfrak{P})\left(0, \eta_{0}\right)
$$

we have

$$
\begin{aligned}
& b-g y-(\operatorname{Re} \mathfrak{P})(x, y)+g d=(b-B)+B-g y-(\operatorname{Re} \mathfrak{P})(x, y)+g d \\
& \quad \geqslant b-g\left(\eta_{0}-d\right)-(\operatorname{Re} \mathfrak{P})\left(0, \eta_{0}\right)>0,
\end{aligned}
$$

for all $-d \leqslant y \leqslant \eta(x)$. For $b$ subject to the constraint (5.5) and close enough to $g\left(\eta_{0}-d\right)+(\operatorname{Re} \mathfrak{P})\left(0, \eta_{0}\right)$, we have $b<B$. These considerations provide us with an estimate of the possible range of values of $B$, in terms of the values of $\mathfrak{p}$.

For a given approximation $b$ of the Bernoulli constant $B$, the corresponding surface elevation $\eta^{b}(x)$ is obtained by the following iterative procedure. Define, for $n \geqslant 1$ and $x \in[0, L / 2]$, the successive approximations

$$
\eta_{n+1}^{b}(x)=\eta_{0}+\int_{0}^{x} \frac{(\operatorname{Im} \mathfrak{P})\left(x^{\prime}, \eta_{n}^{b}\left(x^{\prime}\right)\right)}{b-g \eta_{n}^{b}\left(x^{\prime}\right)-(\operatorname{Re} \mathfrak{P})\left(x^{\prime}, \eta_{n}^{b}\left(x^{\prime}\right)\right)+g d} \mathrm{~d} x^{\prime},
$$



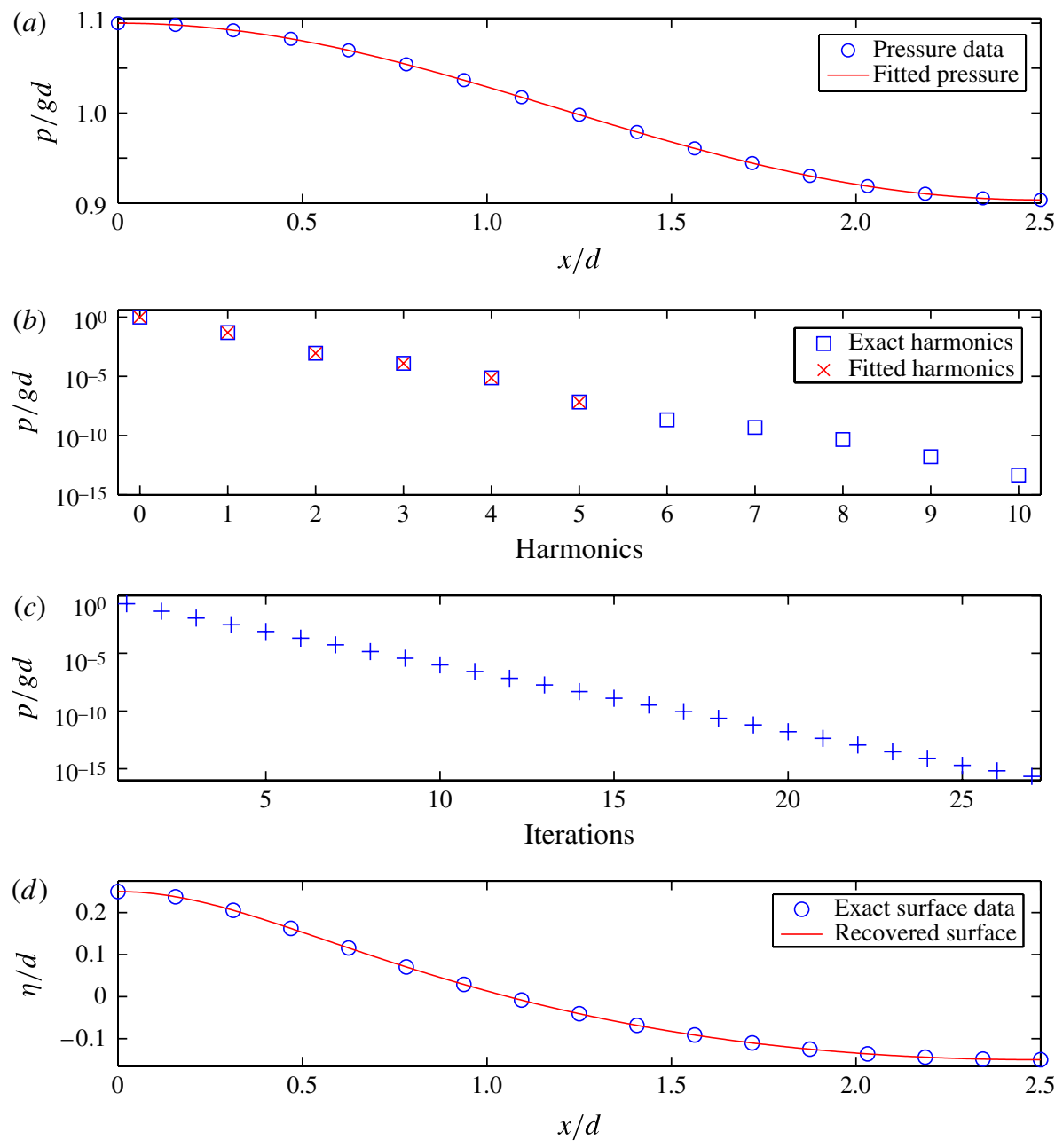

(e) $\quad\left(\times 10^{-5}\right)$

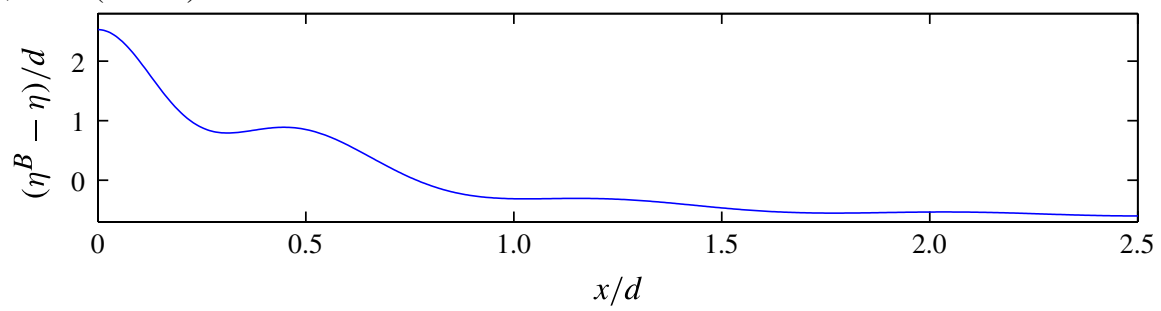

FIGURE 1. (Colour online) Numerical example of the surface reconstruction procedure: $(a)$ pressure at the bed: profile; $(b)$ pressure at the bed: Fourier spectrum; $(c)$ fixed-point iterations of $\eta_{0}$ : absolute errors between two consecutive approximations; $(d)$ recovered free surface: profile; $(e)$ difference between recovered and exact free surfaces.

with initial data $\eta_{0}^{b}(x) \equiv \eta_{0}$. The smoothness and uniform boundedness of the above integrand ensure, by means of the classical Picard-Lipschitz theorem for integral equations (see e.g. Coppel 1965), that the sequence of successive approximations 
converges uniformly on $[0, L / 2]$ to some function $\eta^{b}(x)$. Notice that

$$
v(x, y)>0, \quad x \in(0, L / 2),-d<y<\eta(x),
$$

in view of the considerations by Constantin \& Strauss (2010). In combination with (2.8) and (3.13), this shows that at every fixed $(x, \eta)$ with $x \in(0, L / 2)$, the right-hand side of (3.12) is a strictly increasing function of the parameter $B$. The above iterative procedure yields

$$
\eta^{b_{1}}(x)<\eta^{b_{2}}(x) \leqslant \eta^{B}(x), \quad 0<x \leqslant L / 2,
$$

if $b_{1}<b_{2} \leqslant B$. This shows that there is a unique constant $B$ for which the function $\eta^{B}$ has zero mean. This value of $B$ provides us with the surface wave profile. It can be found by sampling values of $b$ subject to (5.5). Owing to (5.9), we will have $\left\langle\eta^{b}\right\rangle<0$ as long as $b<B$, with $\eta^{b}(0)=\eta_{0}$. Since

$$
\eta^{b}(x)=\eta_{0}+\int_{0}^{x} \frac{(\operatorname{ImP})\left(x^{\prime}, \eta^{b}\left(x^{\prime}\right)\right)}{b-g \eta^{b}\left(x^{\prime}\right)-(\operatorname{Re} \mathfrak{P})\left(x^{\prime}, \eta^{b}\left(x^{\prime}\right)\right)+g d} \mathrm{~d} x^{\prime}, \quad 0 \leqslant x \leqslant L / 2,
$$

taking averages we can state the condition $\left\langle\eta^{B}\right\rangle=0$ equivalently as

$$
\eta_{0}=\frac{2}{L} \int_{0}^{L / 2}\left(x-\frac{L}{2}\right) \frac{(\operatorname{Im} \mathfrak{P})\left(x^{\prime}, \eta^{B}\left(x^{\prime}\right)\right)}{B-g \eta^{B}\left(x^{\prime}\right)-(\operatorname{Re} \mathfrak{P})\left(x^{\prime}, \eta^{B}\left(x^{\prime}\right)\right)+g d} \mathrm{~d} x^{\prime},
$$

obtained by means of integration by parts. In practice, $B$ can be computed by a standard numerical procedure, such as Newton's iterations (see e.g. Press et al. 2007).

\section{Numerical example}

We provide a numerical example of the reconstruction procedure when the pressure at the bed is known numerically at some discrete sampling points. We do not make any attempt to introduce sophisticated numerical techniques. On the contrary, in order to illustrate the efficiency of our recovery procedure, we adopt a layman's straightforward basic numerical implementation of the analytical formulae given above.

We consider an exact surface wave with wave-height/depth ratio equal to 0.25 and wavelength/depth ratio equal to 5 (i.e. $\eta_{0} / d \approx 0.25, k \eta_{0} \approx 0.314$ ). This is thus a wave of significant amplitude in rather deep water, for which the surface reconstruction procedure is a priori difficult (the shallower the water, the easier the reconstruction because of a higher signal/noise ratio). This exact solution is computed numerically by solving the Euler equations (Fenton 1988) and the exact pressure at the bed is thus obtained. We take 32 samples of the bottom pressure equally spaced over one wavelength. These are the pressure 'measurements' we use for the reconstruction. We suppose that $\rho, g$ and $P_{\text {atm }}$ are known parameters, the other parameters having to be computed from the data. We define the accuracy of any computed quantity (i.e. the number of exact digits) by

$$
\text { accuracy } \equiv-\log _{10}\left(\| 1-\text { approximated/exact } \|_{\infty}\right),
$$

where, since we are working in double precision (about sixteen digits), accuracy values higher than sixteen are meaningless and thus rounded down to sixteen.

An analytic approximation of the pressure at the bottom is obtained fitting, via least-square minimization, a fifth-order Fourier polynomial among the pressure data at the bed. (One could also fit the cnoidal profile (4.15) or any other relevant function.) We thus determine accurately the water depth $d$ (cf. the zeroth harmonic in table 1), 


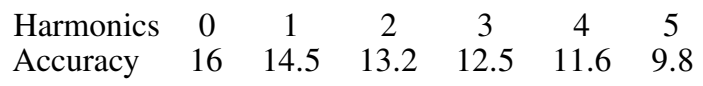

TABLE 1. Accuracy of the harmonics of the fitted fifth-order Fourier polynomial.

as well as the pressure profile (figure 1a) and the main Fourier components (table 1, figure $1 b$ ).

The reconstructed value of $\eta_{0}$ is obtained via straightforward iterations of (3.11), with $\eta_{0}=0$ as initial guess and the fitted fifth-order Fourier polynomial used for $\mathfrak{P}$. The machine precision is reached after 27 iterations with an exponential decay of the error (linear convergence), as shown on figure 1(c). More sophisticated algorithms (e.g. Newton iterations) would reach this precision with fewer iterations. The value of $\eta_{0}$ thus obtained has an accuracy of 4 digits, i.e. the reconstructed amplitude has a relative error of $10^{-4}$ compared to the exact amplitude. Fitting a higher-order Fourier polynomial would give a higher accuracy, provided that there are enough sampled data per period and that these data are precise enough so that the harmonics can be determined with sufficient accuracy.

For a given approximation $b$ of the Bernoulli constant $B$, the reconstruction of $\eta^{b}(x)$ can be obtained as follows. We start with the (constant) initial guess $\eta_{0}^{b}(x)=\eta_{0}$ discretized over 1024 equally spaces nodes. This large number of nodes ensures that the errors are due to the reconstruction procedure, and not to the quadrature formula needed to iterate (5.7). From the $n$th discrete approximation $\eta_{n}^{b}(x)$, the integrand of (5.7) is computed by direct substitution and its mean value is subtracted. An antiderivative with zero mean is then obtained via the fast Fourier transform, thus yielding a new discrete intermediate approximation $\eta_{n+1 / 2}^{b}(x)$. This intermediate approximation is subsequently adjusted so that $\eta_{n+1}^{b}(0)=\eta_{0}$ exactly, i.e.

$$
\eta_{n+1}^{b}(x)=\eta_{n+1 / 2}^{b}(x)-\eta_{n+1 / 2}^{b}(0)+\eta_{0}
$$

The procedure is repeated until convergence, thus giving $\eta^{b}(x)$. Since $\left\langle\eta^{b}\right\rangle \neq 0$ if $b \neq B$, it remains to determine the value of $b=B$ for which $\left\langle\eta^{B}\right\rangle=0$. This equation can be solved for $B$ using standard equation solvers. Here, we use the function fzero of the MATLAB ${ }^{\circledR}$ software. The reconstructed free surface $\eta^{B}(x)$ thus obtained matches the exact one (figure $1 d$ ) with high accuracy (figure $1 e$ ).

This simple example shows that it is possible to reconstruct the free surface with high accuracy, even without using advanced numerical techniques. In practical applications, the accuracy of the recovered surface will depend on the accuracy of the experimental data.

\section{Acknowledgements}

The support of the ERC Advanced Grant 'Nonlinear studies of water flows with vorticity' is acknowledged. The authors are grateful to all three referees for constructive comments and useful suggestions that led to an improved presentation.

\section{REFERENCES}

Abramowitz, M. \& Stegun, I. A. 1965 Handbook of Mathematical Functions. Dover.

Amick, C. J., Fraenkel, L. E. \& Toland, J. F. 1982 On the Stokes conjecture for the wave of extreme form. Acta Mathematica 148, 193-214. 
Baquerizo, A. \& LosadA, M. A. 1995 Transfer function between wave height and wave pressure for progressive waves. Coast. Engng 24, 351-353.

Bishop, C. T. \& Donelan, M. A. 1987 Measuring waves with pressure transducers. Coast. Engng 11, 309-328.

Clamond, D. 1998 Reconstruction du champ de vitesses d'une houle longue. C. R. Acad. Sci. Paris 326, 91-94.

ClAmond, D. 1999 Steady finite amplitude waves on a horizontal seabed of arbitrary depth. J. Fluid Mech. 398, 45-60.

Clamond, D. 2003 Cnoidal-type surface waves in deep water. J. Fluid Mech. 489, 101-120.

Constantin, A. 2006 The trajectories of particles in Stokes waves. Invent. Math. 166, 523-535.

Constantin, A. 2011 Nonlinear water waves with applications to wave-current interactions and tsunamis. In CBMS-NSF Reg. Conf. Ser. Appl. Maths, 81. SIAM.

Constantin, A. 2012 On the recovery of solitary wave profiles from pressure measurements. J. Fluid Mech. 699, 373-384.

Constantin, A. \& Escher, J. 2011 Analyticity of periodic travelling free surface water waves with vorticity. Ann. Maths 173, 559-568.

Constantin, A. \& Strauss, W. 2010 Pressure beneath a Stokes wave. Commun. Pure Appl. Maths 63, 533-557.

Coppel, W. A. 1965 Stability and Asymptotic Behaviour of Differential Equations. D. C. Heath.

Deconinck, B., Henderson, D., Oliveras, K. L. \& VASAn, V. 2011 Recovering the water-wave surface from pressure measurements. In Proc.10th Intl Conf. on WAVES, Vancouver, July 25-29. PIMS (The Pacific Institute for the Mathematical Sciences), pp. 699-702.

Escher, J. \& Schlurmann, T. 2008 On the recovery of the free surface from the pressure within periodic travelling water waves. J. Nonlin. Math. Phys. 15, 50-57.

Fenton, J. D. 1988 The numerical solution of steady water wave problems. Comput. Geosci. 14 (3), 357-368.

Fraenkel, L. E. 2000 An Introduction to Maximum Principles and Symmetry in Elliptic Problems. Cambridge University Press.

Henry, D. 2009 Steady periodic flow induced by the Korteweg-de Vries equation. Wave Motion 46, 403-411.

KuO, Y.-Y. \& CHIU, J.-F. 1994 Transfer function between the wave height and wave pressure for progressive waves. Coast. Engng 23, 81-93.

Окамото, H. \& ShōJI, M. 2001 The Mathematical Theory of Permanent Progressive Water-Waves. World Scientific.

Oliveras, K. L., Vasan, V., Deconinck, B. \& Henderson, D. 2012 Recovering the water-wave profile from pressure measurements. SIAM J. Appl. Maths 72-3, 897-918.

Plotnikov, P. I. \& Toland, J. F. 2002 The Fourier coefficients of Stokes waves. In Nonlinear Problems in Mathematical Physics and Related Topics, I,. pp. 303-315. Kluwer/Plenum.

Press, W. H., Teukolsky, S. A., Vetterling, W. T. \& Flannery, B. P. 2007 Numerical Recipes: The Art of Scientific Computing, 3rd edn. Cambridge University Press.

Spielvogel, E. R. 1970 A variational principle for waves of infinite depth. Arch. Rat. Mech. Anal. 39, $189-205$.

Toland, J. F. 1996 Stokes waves. Topol. Meth. Nonlin. Anal. 7, 1-48.

Tsai, C.-H., Huang, M.-C., Young, F.-J., Lin, Y.-C. \& LI, H. W. 2005 On the recovery of surface wave by pressure transfer function. Ocean Engng 32, 1247-1259.

Varvaruca, E. 2006 Singularities of Bernoulli free boundaries. Commun. Part. Diff. Equ. 31, $1451-1477$. 